\title{
A SINGULAR PERTURBATION RESULT AND ITS APPLICATION TO MATHEMATICAL ECOLOGY
}

\author{
LIGE LI AND A. G. RAMM
}

(Communicated by Barbara L. Keyfitz)

\begin{abstract}
Conditions are given for the solutions of the equation $\varepsilon A u=F(u)$ to converge to the solution of the limit nonlinear equation $F(u)=0$ as $\varepsilon \rightarrow 0$. Applications to existence and uniqueness of the positive solutions of an elliptic predator-prey system are given.
\end{abstract}

\section{INTRODUCTION}

For the Neumann problem $-\Delta u=f(u),\left.\frac{\partial u}{\partial n}\right|_{\partial \Omega}=0$, where $\Omega \subseteq \mathbb{R}^{n}$ is a bounded domain with smooth boundary $\partial \Omega$, it is obvious that there is a nonzero constant solution $u \equiv c$ provided that $c$ is the nonzero equilibrium; i.e., $f(c)=0$. It is also obvious that this is not true for the corresponding homogeneous Dirichlet problem. More interestingly, as has been pointed out by P. DeMottoni and Rothe [2], a certain nonlinear elliptic system in a bounded domain $\Omega \subset \mathbb{R}^{n}$ with smooth boundary $\partial \Omega$,

$$
\begin{aligned}
-\Delta u=h(u)(f(u)-a(v)), \quad-\Delta v & =k(v)(b(u)-g(v)), \\
& \text { in } \Omega, \frac{\partial u}{\left.\partial n\right|_{\partial \Omega}}=0, \frac{\partial v}{\left.\partial n\right|_{\partial \Omega}}=0,
\end{aligned}
$$

where $h, k, f, g, a$, and $b$ are some monotone $C^{1}$ functions, possesses a unique positive solution, which is constant, the positive equilibrium of this system. One cannot expect similar results for positive solutions of the homogeneous Dirichlet problem with boundary data $(u, v)_{\mid \partial \Omega}=(0,0)$. The existence of the (nonzero) constant solution is one of the important features of the Neumann problem.

We prove in this paper that the homogeneous Dirichlet problem

$$
\begin{gathered}
-\Delta u=u(f(u)-a(v)) \quad \text { in } \Omega, \\
-\Delta v=v(b(u)-g(v)) \text { in } \Omega, \quad(u, v)_{\left.\right|_{\partial \Omega}}=(0,0),
\end{gathered}
$$

where $-f, a, b, g$ are monotone increasing $C^{1}$ functions, has the positive solution $(u, v)$ with the property that $(u, v)$ tends to a constant vector, the

Received by the editors August 21, 1989 and, in revised form, February 20, 1990.

1980 Mathematics Subject Classification (1985 Revision). Primary 35J15, 92A17. 
equilibrium state of $(0)$, in any fixed compact subdomain of $\Omega$ as $\Omega \rightarrow \mathbb{R}^{n}$ uniformly in all directions. We assume that $\Omega$ contains the origin.

In equations $(0)$, the functions $u, v$ are the densities of the populations of the prey and the predator, respectively. Biologically, the relative growth rate $f(u)-a(v)$ of the prey is decreasing in $v$ while the relative growth rate $b(u)-g(v)$ of the predator is increasing in $u$. In this sense, the system $(0)$ is a predator-prey model.

In this paper we study an abstract setting for this phenomenon in terms of singular perturbations of some operators including certain generators of $C_{0^{-}}$semigroup of contractions in Hilbert spaces. We also prove the uniqueness of the positive solution to the predator-prey interacting system (0) when the domain $\Omega$ is large. In $\S 2$ a new result in singular perturbation theory is established. This result is used in $\S 3$. A general reference on singular perturbation theory is [7].

\section{SiNGULAR PERTURBATION RESULT}

Consider the following nonlinear equation in a Hilbert space $H$ :

$$
\varepsilon A u=F(u) \text {. }
$$

Let us assume that

$\left(\mathrm{A}_{1}\right) A$ is a closed, densely defined linear operator in $H$ such that

$$
D(A)=D\left(A^{*}\right) \text { and } \operatorname{Re}(A u, u) \geq 0 \text { for } \forall u \in D(A)
$$

where $A^{*}$ is the adjoint operator to $A$.

$\left(\mathrm{A}_{2}\right) \quad F$ is a continuously Fréchet differentiable nonlinear operator with an isolated zero $w_{+}, F\left(w_{+}\right)=0$; i.e., in a neighborhood $B_{\delta}\left(w_{+}\right)=\{u$ : $\left.\left\|w_{+}-u\right\| \leq \delta\right\}$ of $w_{+}$there are no other zeros of $F$. Denote $B=$ $-F^{\prime}\left(w_{+}\right)$. Assume

$$
\operatorname{Re}(B f, f) \geq c\|f\|^{2}, \quad \forall f \in H,
$$

where $c$ is a positive constant.

Theorem 1. Given the assumptions $\left(\mathrm{A}_{1}\right)$ and $\left(\mathrm{A}_{2}\right)$, equation (1) has a solution in a neighborhood $B_{\delta}\left(w_{+}\right)$of $w_{+}$for all $0 \leq \varepsilon \leq \varepsilon_{0}(\delta)$ provided that $\delta>0$ is sufficiently small and $\varepsilon_{0}(\delta)$ is suitably chosen. This solution $u_{\varepsilon}$ is unique in $B_{\delta}\left(w_{+}\right)$and $\left\|w_{+}-u_{\varepsilon}\right\| \rightarrow 0$ as $\varepsilon \rightarrow 0$.

Proof. Since $F\left(w_{+}\right)=0$ and $F$ is Fréchet differentiable, one may write equation (1) as

$$
(\varepsilon A+B) u=B w+\beta\left(u-w_{+}\right)
$$

in a neighborhood of $w_{+}$where $\|\beta(v)\|=o(\|v\|)$ as $v \rightarrow 0$. Let $C_{\varepsilon}$ denote the operator $\varepsilon A+B$. Then

$$
\operatorname{Re}\left(C_{\varepsilon} u, u\right) \geq c\|u\|^{2}
$$


where $c$ is the constant given in (3). Let $N(A)=\{u \mid A u=0\}, R(A)=\{f \mid f=$ $A u\}$ be the null space and the range of $A$. It follows from (2) and (5) that $N\left(C_{\varepsilon}\right)=\{0\}$ and $N\left(C_{\varepsilon}^{*}\right)=\{0\}$. Since $\overline{R\left(C_{\varepsilon}\right)} \oplus N\left(C_{\varepsilon}^{*}\right)=H$ it follows that $\overline{R\left(C_{\varepsilon}\right)}=H$. Using (5) and the fact that $C_{\varepsilon}=\varepsilon A+B$ is closed, one checks that $\overline{R\left(C_{\varepsilon}\right)}=R\left(C_{\varepsilon}\right)$, that $(\varepsilon A+B)^{-1}$ is everywhere defined on $H$ and

$$
\left\|(\varepsilon A+B)^{-1}\right\| \leq c^{-1}
$$

for all $\varepsilon \geq 0$.

The first step is to show that, for all $f \in H$ one has

$$
\left\|(\varepsilon A+B)^{-1} B f-f\right\| \rightarrow 0 \text { as } \varepsilon \rightarrow+0 .
$$

Note that $f$ may not be in $D(A)$, and, in general, the operator $(\varepsilon A+B)^{-1} B$ may not converge to $I$ in the operator norm. However, since $D(A)$ is dense in $H$, there is a $w \in D(A)$ such that $\|w-f\|<\eta$ where $\eta$ is an arbitrary small number. One has

$$
\begin{aligned}
\left\|(\varepsilon A+B)^{-1} B f-f\right\|= & \left\|(\varepsilon A+B)^{-1} B w-w+(\varepsilon A+B)^{-1} B(f-w)+w-f\right\| \\
\leq & \left\|(\varepsilon A+B)^{-1}(B+\varepsilon A-\varepsilon A) w-w\right\| \\
& +\left\|(\varepsilon A+B)^{-1}\right\|\|B(f-w)\|+\|f-w\| \\
\leq & c^{-1} \varepsilon\|A w\|+c^{-1}\|B f-B w\|+\|f-w\| \leq \theta(\varepsilon+\eta)
\end{aligned}
$$

where $\theta>0$ is a constant. Since $\eta$ is arbitrarily small, this proves (7).

The second step is to apply the contraction mapping principle to (4). Equation (4) can be rewritten as

$$
u=Q_{\varepsilon} u:=(\varepsilon A+B)^{-1} B w_{+}+(\varepsilon A+B)^{-1} \beta\left(u-w_{+}\right) .
$$

Let us check that (1) the operator $Q_{\varepsilon}$ maps $B_{\delta}\left(w_{+}\right)$into itself for sufficiently small $\delta$ and $\varepsilon$ and (2) $Q_{\varepsilon}$ is a contraction mapping on $B_{\delta}\left(w_{+}\right)$.

Let $u \in B_{\delta}\left(w_{+}\right)$. Then $\left\|Q_{\varepsilon} u-w_{+}\right\| \leq\left\|(\varepsilon A+B)^{-1} B w_{+}-w_{+}\right\|+c^{-1}\left\|\beta\left(u-w_{+}\right)\right\|$. Statement (1) follows from (7) and the estimate for $\beta$ given below (4). Let $u_{1}$ and $u_{2}$ belong to $B_{\delta}\left(w_{+}\right)$. One has

$$
\begin{aligned}
\left\|Q_{\varepsilon} u_{1}-Q_{\varepsilon} u_{2}\right\| & \leq c^{-1}\left\|\beta\left(u_{1}-w_{+}\right)-\beta\left(u_{2}-w_{+}\right)\right\| \\
& \leq c^{-1} \alpha(\delta)\left\|u_{1}-u_{2}\right\|,
\end{aligned}
$$

where $\alpha(\delta) \rightarrow 0$ as $\delta \rightarrow 0$ because, by the assumption, $F$ is continuously Fréchet differentiable. This proves statement (2). Theorem 1 is proved.

A general result which gives a necessary and sufficient condition for a solution of an operator equation to depend continuously (or smoothly) on the parameter is given in [9].

Remark 1. We give another version of Theorem 1 under different assumptions $\left(\mathrm{A}_{1}^{\prime}\right)$ and $\left(\mathrm{A}_{2}^{\prime}\right)$ :

$\left(\mathrm{A}_{1}^{\prime}\right)-A$ is a generator of $C_{o}$-semigroup of contractions on $H$. 
$\left(A_{2}^{\prime}\right)$ Assume $\left(A_{2}\right)$ holds and

$$
\operatorname{Re}\left(B^{-1} A f, f\right) \geq 0 \text { for } \forall f \in D(A) .
$$

Note that $\left(\mathrm{A}_{1}\right)$ implies $\left(\mathrm{A}_{1}^{\prime}\right)$ (see $\left.[8, \S 1.4]\right)$. Since $B$ is bounded and $\operatorname{Re}(B f, f) \geq c\|f\|^{2}$ for all $f \in H$, one concludes that $R(B)=H$ and $B^{-1}$ is a bounded operator with $D\left(B^{-1}\right)=H$.

Claim. The conclusion of Theorem 1 holds under assumptions $\left(A_{1}^{\prime}\right)$ and $\left(A_{2}^{\prime}\right)$. Proof. Note that the following inequality

$$
\operatorname{Re}\left(B^{-1} u, u\right) \geq c_{1}\|u\|^{2}, \quad \forall u \in H,
$$

where $c_{1}$ is a positive constant, follows easily from (3) and the boundedness of $B$. By the assumptions $\left(\mathrm{A}_{2}^{\prime}\right),(9)$, and $\left(9^{\prime}\right)$ we conclude that $-B^{-1} A=$ $B^{-1}(-A)$ is a generator of $C_{o}$-semigroup of contractions because $-A$ is. This is true because of the following result (see [3, Theorem $2 \mathrm{~B}(\mathrm{i})]$ ):

Let $G$ be a generator of $C_{o}$-semigroup of contractions in a Hilbert space $H$ and an operator $C$ be strongly accretive; i.e., $\operatorname{Re}(C u, u) \geq \eta\|u\|^{2}, \eta=$ const $>0$. Then $C G$ is a generator of $C_{o}$-semigroup of contractions if and only if $\operatorname{Re}(C G u, u) \leq 0$ for all $u \in D(C G)$.

In our case, $G=-A$ and $C=B^{-1}$. Thus $\varepsilon^{-1}$ is in the resolvent set of $-B^{-1} A$ for any $\varepsilon^{-1}>0$, and therefore we may write

$$
\begin{aligned}
(\varepsilon A+B)^{-1} & =\left[B\left(\varepsilon B^{-1} A+I\right)\right]^{-1}=\left(I-\varepsilon\left(-B^{-1} A\right)\right)^{-1} B^{-1} \\
& =\varepsilon^{-1}\left[\varepsilon^{-1}-\left(-B^{-1} A\right)\right]^{-1} B^{-1} .
\end{aligned}
$$

This implies that $(\varepsilon A+B)^{-1}$ is defined everywhere. Formula (7) is equivalent to

$$
\left\|\varepsilon^{-1}\left[\varepsilon^{-1} I-\left(-B^{-1} A\right)\right]^{-1} f-f\right\| \rightarrow 0 \text { as } \varepsilon \rightarrow 0, \forall f \in H
$$

(see, for example [8, p. 9, Lemma 3.2]). Therefore the proof of Theorem 1 remains valid under the assumptions $\left(A_{1}^{\prime}\right)$ and $\left(A_{2}^{\prime}\right)$.

In some mathematical ecology models the operator $B=-F^{\prime}\left(w_{+}\right)$is an isomorphism of $H$ onto $H$ and the result in Remark 1 is useful.

In many applications the operator $B=-F^{\prime}\left(w_{+}\right)$is not accretive. It satisfies a weaker assumption: $\sigma\left(F^{\prime}\left(w_{+}\right)\right) \subseteq\{z: z \subseteq \mathbb{C}, \operatorname{Re} z \leq-\tilde{c}<0\}, \tilde{c}=$ const , and the operator $-A$ is often a generator of an analytic compact semigroup.

We are interested in the problem $\varepsilon A w=F(w)$ in the space $H=H_{1} \oplus H_{1}$ where $H_{1}$ is a Hilbert space, $w=\left(\begin{array}{l}u \\ v\end{array}\right), u, v \in H_{1}$, and $A=\left(\begin{array}{cc}A_{1} & 0 \\ 0 & A_{1}\end{array}\right)$ where $A_{1}: H_{1} \rightarrow H_{1}$ satisfies the assumption:

$\left(\mathrm{A}_{3}\right)-A_{1}$ is a generator of an analytic compact semigroup of contractions on $H_{1}$ with the spectrum $\sigma\left(A_{1}\right)$ consisting of countably many eigenvalues $\left\{\lambda_{n}\right\}$ and the corresponding eigenvectors $\phi_{n}$ form an orthonormal basis of $H_{1}$ : $\left\langle\phi_{n}, \phi_{m}\right\rangle=\delta_{n m}, n, m=1,2, \ldots$. 
It is known that the spectrum $\sigma\left(-A_{1}\right)$ of a generator $-A_{1}$ of a compact semigroup contains at most countably many eigenvalues [8]. In $\S 3$ the operator $A$ is the Dirichlet Laplacian in a bounded domain, which is a generator of an analytic and compact semigroup [4].

The following assumption is imposed on the operator $B$ :

$\left(\mathrm{A}_{4}\right)-B=F^{\prime}\left(w_{+}\right)$is a bounded linear operator on $H=H_{1} \oplus H_{1}$ such that the spectrum $\sigma\left(F^{\prime}\left(w_{+}\right)\right) \subset\{z: z \in \mathbb{C}, \operatorname{Re} z \leq-\tilde{c}<0\}$. Furthermore, we assume that for any element $a \in H_{1}, B:\langle a\rangle \oplus\langle a\rangle \rightarrow\langle a\rangle \oplus\langle a\rangle$, where $\langle a\rangle$ denotes the 1-dimensional space generated by $a$ so that $\langle a\rangle=\{\lambda a\}, \lambda \in \mathbb{C}$.

Example. Let $B=\left(\begin{array}{ll}\alpha & \beta \\ \gamma & \delta\end{array}\right)$ where $\alpha, \beta, \gamma, \delta \in \mathbb{C}$; then $B:\langle a\rangle \oplus\langle a\rangle \rightarrow\langle a\rangle \oplus\langle a\rangle$.

We now prove the following theorem:

Theorem 2. Given the assumptions $\left(\mathrm{A}_{3}\right)$ and $\left(\mathrm{A}_{4}\right)$, the conclusion of Theorem 1 holds.

Proof. We first prove that there is a constant $c_{1}>0$ such that $\sigma(\varepsilon A+B) \subseteq$ $\left\{z: z \in \mathbb{C}, \operatorname{Re} z \geq c_{1}>0\right\}$. Consider the Cauchy problem on $H=H_{1} \oplus H_{1}:$

$$
\frac{d w_{\varepsilon}}{d t}=-(\varepsilon A+B) w_{\varepsilon}, \quad w_{\varepsilon}(0)=w_{0} \in D(A)
$$

where $B=-F^{\prime}\left(w_{+}\right), w_{\varepsilon}=\left(u_{\varepsilon}, v_{\varepsilon}\right), w_{0}=\left(u_{0}, v_{0}\right), u_{0}, v_{0} \in D\left(A_{1}\right)$ are arbitrary, $w_{0} \in D(A), A=\left(\begin{array}{cc}A_{1} & 0 \\ 0 & A_{1}\end{array}\right)$. Let $u_{\varepsilon}(t)=\sum_{n=1}^{\infty} a_{n}^{\varepsilon}(t) \phi_{n}, v_{\varepsilon}(t)=$ $\sum_{n=1}^{\infty} b_{n}^{\varepsilon}(t) \phi_{n}$ where $\phi_{n}$ are the eigenvectors of $A_{1}$ in $H_{1}$. Equating the coefficients in front of $\phi_{n}$ in $(*)$, one obtains the following equation:

$$
\frac{d}{d t}\left(a_{n}^{\varepsilon}(t) \phi_{n}, b_{n}^{\varepsilon}(t) \phi_{n}\right)^{T}=\left(F^{\prime}\left(w_{+}\right)-\varepsilon \lambda_{n} I\right)\left(a_{n}^{\varepsilon} \phi_{n}, b_{n}^{\varepsilon} \phi_{n}\right)^{T},
$$

where $T$ stands for the transpose. This equation can be written as

$$
\frac{d}{d t}\left(a_{n}^{\varepsilon}(t)\left(\begin{array}{c}
\phi_{n} \\
0
\end{array}\right)+b_{n}^{\varepsilon}(t)\left(\begin{array}{c}
0 \\
\phi_{n}
\end{array}\right)\right)=\left(F^{\prime}\left(w_{+}\right)-\varepsilon \lambda_{n} I\right)\left[a_{n}^{\varepsilon}\left(\begin{array}{c}
\phi_{n} \\
0
\end{array}\right)+b_{n}^{\varepsilon}\left(\begin{array}{c}
0 \\
\phi_{n}
\end{array}\right)\right] .
$$

Denote by $F_{n}$ the restriction of $-F^{\prime}\left(w_{+}\right)$to the set $\left(\begin{array}{l}\lambda \phi_{n} \\ \mu \phi_{n}\end{array}\right), \lambda, \mu \in \mathbb{C}$. By the assumption $\left(\mathrm{A}_{4}\right)$, this set is invariant for $B . F_{n}$ can be represented by a matrix $\left(\begin{array}{c}\alpha_{n} \beta_{n} \\ \gamma_{n} \delta_{n}\end{array}\right)$ where $\alpha_{n}, \beta_{n}, \gamma_{n}$, and $\delta_{n}$ are numbers.

Therefore one has

$$
\frac{d}{d t}\left(\begin{array}{c}
a_{n}^{\varepsilon}(t) \\
b_{n}^{\varepsilon}(t)
\end{array}\right)=\left(F_{n}-\varepsilon \lambda_{n} I\right)\left(\begin{array}{l}
a_{n}^{\varepsilon}(t) \\
b_{n}^{\varepsilon}(t)
\end{array}\right) .
$$

Note that $\operatorname{Re} \lambda_{n} \geq 0$, because $-A_{1}$ is a generator of a semigroup of contractions (see [9]). Since $\sigma\left(F^{\prime}\left(w_{+}\right)\right) \subset\{z: z \in \mathbb{C}, \operatorname{Re} z \leq-\tilde{c}<0\}$ one has $\sigma\left(F_{n}\right) \subset\{z: z \in \mathbb{C}, \operatorname{Re} z \leq-\tilde{c}<0\}$. Thus $\sigma\left(F_{n}-\varepsilon \lambda_{n} I\right) \subset\{z: \operatorname{Re} z \leq-\tilde{c}<$ $0\}$. Therefore $\left\|\left(\begin{array}{l}a_{n}^{\varepsilon}(t) \\ b_{n}^{2}(t)\end{array}\right)\right\|:=\left[\left(a_{n}^{\varepsilon}(t)\right)^{2}+\left(b_{n}^{\varepsilon}(t)\right)^{2}\right]^{1 / 2} \leq M \exp (-\tilde{c} t)\left\|\left(\begin{array}{l}a_{n}^{\varepsilon}(0) \\ b_{n}^{\varepsilon}(0)\end{array}\right)\right\|$, where $a_{n}^{\varepsilon}(0)=\left\langle u_{0}, \phi_{n}\right\rangle, b_{n}^{\varepsilon}(0)=\left\langle v_{0}, \phi_{n}\right\rangle$, and $M, \tilde{c}>0$ are independent of $n$. Parseval's equality yields $\|w\| \leq M \exp (-\tilde{c} t)\left\|w_{0}\right\|$. Thus $\left\|P_{\varepsilon}(t)\right\| \leq M \exp (-\tilde{c} t)$ 
where $P_{\varepsilon}(t)$ is the semigroup generated by the operator $-(\varepsilon A+B)$. One has $\lim _{t \rightarrow \infty} t^{-1} \ln \left\|P_{\varepsilon}(t)\right\|=\max \{\operatorname{Re} \lambda \mid \lambda \in \sigma(-(\varepsilon A+B))\}$ and $\sigma(-(\varepsilon A+B)) \subset\{z:$ $\operatorname{Re} z \leq-\tilde{c}<0\}$. Therefore $0 \notin \sigma(-(\varepsilon A+B))$. Note that $-(\varepsilon A+B)$ is a generator of an analytic semigroup because $-A$ is and $B$ is bounded. Therefore one can use the estimate for the norm of the resolvent $(\lambda+\varepsilon A+B)^{-1}$ for $\lambda=0$ (see [8, p. 61]) and get $\left\|(\varepsilon A+B)^{-1}\right\| \leq$ const, i.e., estimate (6) holds. The argument given below formula (6) in the proof of Theorem 1 remains valid and this proves Theorem 2.

\section{Applications}

In this section we study the uniqueness, existence, and stability of the positive solutions of a predator-prey system as the domain $\Omega$, on which the solution is defined, expands. Consider the elliptic predator-prey system (0) .

Assume that the functions $-f, a, g$, and $m$ are strictly increasing $C^{1}$ functions. Assume further that

$\left(\mathrm{H}_{1}\right)$. $\left|f^{\prime}(x)\right| \geq L>0$ for $x>0$ where $L$ is a constant, $f(\gamma)=0$ for some $\gamma>0$ (the carrying capacity), $a(0)=0$, and $g(0)<m(0)<g(\gamma)$.

In biological applications, $\left(\mathrm{H}_{1}\right)$ indicates that the model is logistic. The relation $g(0)<m(0)$ means that the predator dies in the absence of prey species (see [5]). One can show (see [5, p. 151]) that the positive solutions of equations (0) satisfy a priori bounds $0 \leq u<\gamma, 0 \leq v<m^{-1}(g(\gamma))$ with $\gamma$ defined in assumption $\left(\mathrm{H}_{1}\right)$. Let $M=\max \left(\gamma, m^{-1} g(\gamma)\right)$. Then $0 \leq u, v<M$. Note that these a priori bounds depend only on the nonlinearities $f, a, m$, and $g$. $\left(\mathrm{H}_{2}\right)$. Let $C_{1}=m^{-1}(g(\gamma))$. Assume $a\left(C_{1}\right)<f(0)$.

From the assumption $m(0)<g(\gamma)$ in $\left(\mathrm{H}_{1}\right)$ it follows that $C_{1}>0$. Since $f^{-1}(a(v))$ is decreasing, $g^{-1}(m(v))$ is increasing, and $0<g^{-1}(m(0))<\gamma=$ $f^{-1}(a(0))$, one concludes that system (0) has a unique positive equilibrium $w_{+}=(\hat{u}, \hat{v})$, where $\hat{u}>0, \hat{v}>0$ are constants such that $f(\hat{u})-a(\hat{v})=0$, $g(\hat{u})-m(\hat{v})=0$.

Given the assumptions $\left(\mathrm{H}_{1}\right)$ and $\left(\mathrm{H}_{2}\right)$, we prove the uniqueness of the positive solutions to system $(0)$ for sufficiently large domains $R \Omega:=\{x: x=$ $R y, y \in \Omega\}, R>0$, using Theorem 2 and a result from [6].

Let $H=L^{2}(\Omega) \oplus L^{2}(\Omega), w=(u, v), A: D(A) \rightarrow H$ is the operator given by the differential expression $A=\left(\begin{array}{cc}-\Delta & 0 \\ 0 & -\Delta\end{array}\right)$ and the homogeneous Dirichlet boundary conditions, $D(A)=\dot{H}^{2}(\Omega) \times \dot{H}^{2}(\Omega)$, where $\dot{H}^{2}(\Omega)$ is the usual Sobolev space $\dot{H}^{2}(\Omega)=H^{2}(\Omega) \cap \dot{H}^{1}(\Omega)$, and $\dot{H}^{1}(\Omega)$ is the closure in the norm of $H^{1}(\Omega)$ of $C_{0}^{\infty}(\Omega)$.

Let $F(w)=\left(\begin{array}{l}u(f(u)-a(v)) \\ v(g(u)-m(v))\end{array}\right)$ and $w_{+}=(\hat{u}, \hat{v})$. One has

$$
F^{\prime}\left(w_{+}\right)=\left(\begin{array}{ll}
\hat{u} f^{\prime}(\hat{u}), & -\hat{u} a^{\prime}(\hat{v}) \\
\hat{v} g^{\prime}(\hat{u}), & -\hat{v} m^{\prime}(\hat{v})
\end{array}\right) .
$$


The operator $F: w \rightarrow F(w)$ is Fréchet differentiable in $H$ if the matrixfunction $F^{\prime}(w)$ is uniformly bounded for all $w \in R^{2}$. In the biological problem under consideration, the behavior of the functions $f, a, g$, and $m$ outside of a sufficiently large region, that is, outside of a region $|u|+|v| \leq \rho$, where $\rho>0$ is a sufficiently large number, can be arbitrary since the solutions to (0) are a priori bounded. Therefore one can assume that $F^{\prime}(w)$ is a uniformly bounded matrix function and the operator $F$ is Fréchet differentiable in $H$ at the element $w_{+}$.

Let $\mu_{1}, \mu_{2}$ be the eigenvalues of matrix $F^{\prime}\left(w_{+}\right)$. One can check that $\operatorname{Re} \mu_{1}<$ 0 and $\operatorname{Re} \mu_{2}<0$. Thus, the spectrum $\sigma\left(F^{\prime}\left(w_{+}\right)\right) \subset\left\{z: z \in \mathbb{C}, \operatorname{Re} z \leq-c_{2}<\right.$ $0\}$ for some constant $c_{2}>0$.

It is clear that for the operators $A$ and $B=-F^{\prime}\left(w_{+}\right)$, the assumptions $\left(\mathbf{A}_{3}\right)$ and $\left(\mathrm{A}_{4}\right)$ of Theorem 2 are satisfied. To investigate system $(0)$ over a large domain in $\mathbf{R}^{n}$, we use a change of variables. Let $R \Omega$ be as above. Define $\bar{u}(\bar{x}):=u(x)$, and $\bar{v}(\bar{x}):=v(x)$ for $x \in R \Omega$ where $\bar{x}=x / R, \bar{x} \in \Omega$. Then the functions $u$ and $v$ satisfy the equations

$$
\begin{gathered}
\Delta u+u(f(u)-a(v))=0 \quad \text { in } R \Omega, \\
\Delta v+v(g(u)-m(v))=0 \quad \text { in } R \Omega, \quad(u, v)_{\left.\right|_{\partial(R \Omega)}}=(0,0)
\end{gathered}
$$

if and only if the functions $\bar{u}, \bar{v}$ satisfy the equations

$$
\begin{array}{cl}
R^{-2} \Delta \bar{u}+\bar{u}(f(\bar{u})-a(\bar{v}))=0 & \text { in } \Omega, \\
R^{-2} \Delta \bar{v}+\bar{v}(g(\bar{u})-m(\bar{v}))=0 \quad \text { in } \Omega, & (\bar{u}, \bar{v})_{\left.\right|_{\partial \Omega}}=(0,0) .
\end{array}
$$

One could assume different diffusion rates in (11), divide by these rates, and reduce the problem to the one with equal diffusion rates. Set $\varepsilon=R^{-2}$. Then the equations in (12) can be rewritten as $\varepsilon A \bar{w}=F(\bar{w})$, where $\bar{w}=\left(\frac{\bar{u}}{v}\right)$.

The operators $A$ and $F$ satisfy the assumptions in Theorem 2 , and this theorem implies the existence and uniqueness of a solution $w_{\varepsilon}$ to (12) in a small neighborhood of $w_{+}$if $\varepsilon=R^{-2}$ is sufficiently small. In other words, in a sufficiently large domain $R \Omega$, system (11) has a solution $\left(u_{\varepsilon}, v_{\varepsilon}\right)$ in a neighborhood of $w_{+}=(\hat{u}, \hat{v})$. Since the solutions $\left(u_{\varepsilon}, v_{\varepsilon}\right)$ converge in $H$ to $w_{+}=(\hat{u}, \hat{v})$, one concludes, using Theorem 2 , that for all sufficiently large $R$, system (11) has a unique solution $\left(u_{\varepsilon}, v_{\varepsilon}\right)$.

We have proved the following:

Proposition 1. If $\left(\mathrm{H}_{1}\right)$ and $\left(\mathrm{H}_{2}\right)$ hold and $R>0$ is sufficiently large, then problem (11) has a unique solution in a neighborhood of the equilibrium $w_{+}$.

The uniqueness result in this proposition is of a local nature. To obtain a global uniqueness result we need Theorem 3 from [6] which says that hypotheses $\mathrm{H}_{1}$ and $\mathrm{H}_{2}$ imply that there exist positive solutions of equation (12), and for any such positive solution $\left(\bar{u}_{R}, \bar{v}_{R}\right)$ the following relations hold:

$$
\left\|\bar{u}_{R}-\hat{u}\right\|_{L^{p}(\Omega)} \rightarrow 0,\left\|\bar{v}_{R}-\hat{v}\right\|_{L^{p}(\Omega)} \rightarrow 0 \quad \text { as } R \rightarrow \infty \text { for all } p>1 .
$$


Since Theorem 2 deals with Hilbert spaces, one uses the above result with $p=$ 2 , and concludes that $\left(\bar{u}_{R}, \bar{v}_{R}\right)$ belongs to an arbitrarily small neighborhood of $w_{+}=(\hat{u}, \hat{v})$ in $H=L^{2}(\Omega) \oplus L^{2}(\Omega)$, provided that $R>0$ is sufficiently large. Combining this result with the local uniqueness of the solution to (11) established in Proposition 1, we conclude that the positive solution of (11) and (12) is globally unique for all sufficiently large $R$.

Theorem 3. Under the assumptions $\left(\mathrm{H}_{1}\right)$ and $\left(\mathrm{H}_{2}\right)$, the positive solution to system (11) exists and is (globally) unique for all sufficiently large domains $R \Omega$.

\section{ACKNOWLEDGMENTS}

The authors thank the referee for helpful suggestions. The second author thanks NSF and ONR for support. The first author thanks E. N. Dancer for a helpful remark.

\section{REFERENCES}

1. E. D. Conway and J. A. Smoller, Diffusion and the predator-prey interaction, SIAM J. Appl. Math. 33 (1977), 673-686.

2. P. DeMottoni and F. Rothe, Convergence to homogeneous equilibrium states for generalized Volterra-Lotka systems, SIAM J. Appl. Math. 37 (1979), 648-663.

3. K. Gustafson and G. Lumer, Multiplicative perturbation $f$ semigroup generators, Pacific $\mathbf{J}$. Math. 41 (1972), 731-742.

4. T. Kato, Perturbation theory for linear operators, Springer-Verlag, Berlin, 1984.

5. L. Li, Coexistence theorems of steady state for predator-prey interacting systems, Trans. Amer. Math. Soc. 305 (1988), 143-166.

6. L. Li and M. R. Lloyd, A numerical behavior of positive solution to elliptic predator-prey systems over large domain (Ed. by R. Aftabizadeh), Differential Equations and Applications, vol. 2, Ohio Univ. Press, 1989, pp. 124-132.

7. S. Lomov, Introduction into a general theory of singular perturbations, Nauka, Moscow, 1981. (Russian)

8. A. Pazy, Semigroups of linear operators and applications to partial differential equations, Springer-Verlag, New York, 1983.

9. A. G. Ramm, Some theorems on operator equations depending on a parameter in Banach spaces, Doklady Acad. Sci. Azerb. USSR 22 (1966), 3-6.

Department of Mathematics, Kansas State University, Manhattan, Kansas 66506

E-mail address, A. G. Ramm: ramm@ksuvm.bitnet 